\title{
Cancer Therapy Based on Nanomaterials and Nanocarrier Systems
}

\author{
Weili Qiao, Bochu Wang, Yazhou Wang, Lichun Yang, Yiqiong Zhang, and Pengyu Shao \\ College of Bioengineering, Chongqing University, Chongqing 400030, China \\ Correspondence should be addressed to Bochu Wang, wangbc2000@126.com
}

Received 30 September 2009; Revised 1 December 2009; Accepted 21 January 2010

Academic Editor: Chao Lin

Copyright () 2010 Weili Qiao et al. This is an open access article distributed under the Creative Commons Attribution License, which permits unrestricted use, distribution, and reproduction in any medium, provided the original work is properly cited.

Targeted delivery of drug molecules to tumor tissue is one of the most interesting and challenging endeavors faced in pharmaceutical field, due to the critical and pharmacokinetically specific environment that exists in tumor. Over these years, cancer targeting treatment has been greatly improved by new tools and approaches based on nanotechnology. The review firstly introduces the specific physical and chemical properties of a serial of nanomaterials, such as nanoparticles, micelles, dendrimers, carbon nanotubes, quantum dots, and nanofibers. It then places great emphasis on their application in the field of cancer therapy when they are used as nanocarrier systems. Based on the current status, the paper further discusses the unsolved problems and makes a perspective for the future prospects of the nanocarrier systems.

\section{Introduction}

Cancer occurs at a molecular level when multiple subsets of genes undergo genetic alterations, either activation of oncogenes or inactivation of tumor suppressor genes. Then malignant proliferation of cancer cells, tissue infiltration, and dysfunction of organs will appear [1]. Tumor tissues are characterized with active angiogenesis and high vascular density which keep blood supply for their growth, but with a defective vascular architecture. Combined with poor lymphatic drainage, they contribute to what is known as the enhanced permeation and retention (EPR) effect $[2,3]$. Tumor genes are not stable with their development and often show genovariation. The inherent complexity of tumor microenvironment and the existence of P-glycoprotein (Pgp) usually act as barriers to traditional chemotherapy by preventing drug from reaching the tumor mass. Meanwhile, delivery of the therapeutic agents in vivo shares physiological barriers, including hepatic and renal clearance, enzymolysis and hydrolysis, as well as endosomal/lysosomal degradation $[4,5]$. In addition, the efficiency of anticancer drugs is limited by their unsatisfactory properties, such as poor solubility, narrow therapeutic window, and intensive cytotoxicity to normal tissues, which may be the causes of treatment failure in cancer [6].
Accordingly, there is a great need for new therapeutic strategies capable of delivering chemical agents and other therapeutic materials specifically to tumor locations [7]. With the development of nanotechnology, the integration of nanomaterials into cancer therapeutics is one of the rapidly advancing fields. It can revolutionize the treatment of cancer. Nanotechnology is the creation and utilization of materials, devices, and systems through the control of matter on the nanometer (1 billionth of a meter) scale [8]. Nanocarrier systems can be designed to interact with target cells and tissues or respond to stimuli in well-controlled ways to induce desired physiological responses. They represent new directions for more effective diagnosis and therapy of cancer [9]. Therefore, this pape discusses the characteristics of various nanotechnology-based drug delivery systems in details, including nanoparticles, micelles, carbon nanotubes, dendrimers, quantunm dots, and nanofibers, and with more emphasis on their applications in cancer therapy.

\section{Different Nanotechnology-Based Nanocarrier Systems}

Based on nanotechnology, nanocarriers synthesized from organic and inorganic materials have been developed, such 
as nanoparticles, micelles, carbon nanotubes, dendrimers, quantunm dots, and nanofibers $[10,11]$ (Figure 1). They have shown great potential in cancer therapy by enhancing the performance of medicines and reducing systemic side effect in order to gain therapeutic efficiency.

2.1. Polymeric Nanoparticles. Polymeric nanopaticles are particles of less than $1 \mu \mathrm{m}$ diameter that are prepared from natural or synthetic polymers. Depending on the methods of preparation, nanoparticles can be obtained with different properties and different release characteristics by forming matrix-type or reservoir-type structure, named nanospheres or nanocapsules [12]. They have been considered as the promising carriers for drug delivery because they can improve the specificity of action of drugs by changing their tissue distribution and pharmacokinetics [13]. Polymeric nanoparticles have played pivotal roles in delivering antitumor drugs in a targeted manner to the malignant tumor cells, thereby reducing the systemic toxicity and increasing their therapeutic efficacy. Due to the reticulo-endothelial system (RES) and the effect of enhanced permeation and retention (EPR), nanoparticles can be formulated for passive delivery to the lymphatic system, brain, arterial walls, lungs, liver, spleen, or made for long-term systemic circulation $[2,13,14]$. Importantly, the critical feature of polymeric nanoparticles as drug carriers is that they are amenable to surface functionalization for active targeting to tumor tissues or cells and for stimulus-responsive controlled release of drug [15]. Active targeting of nanoparticles to action sites is based on the pathological state of tumor tissues, such as the angiogenesis and the overexpressed receptors. Thus, varieties of researchers have focused on formulating multifunctional nanoparticles to improve the effectiveness of drug delivery and therapy [15].

The receptor-mediated endocytosis (RME) reveals the selective recognition, high-affinity binding, and immediate internalization for the ligands at a cellular level [16]. For this reason, various targeting moieties can be attached to the polymer backbone, which will act as a secondary uptake mechanism following EPR-based primary accumulation [3, 17] (Figure 2). Liang et al. developed paclitaxelloaded nanoparticles with galactosamine conjugated on for targeting to liver cancer cells. The prepared nanoparticles appeared most efficient in reducing the size of the tumor when injected into hepatoma-tumor-bearing nude mice, through the specific interaction between galactosamine and asialoglycoprotein receptors [18]. Thermosensitive magnetoliposomes (TMs) encapsulated with methotrexate (MTX) prepared by reverse-phase evaporation can achieve a good magnetic targeting effect and rapid drug release in response to hyperthermia, which implies their great potential in cancer therapy [19].

2.2. Micelles. Polymeric micelles are usually formed into core-shell structures by spontaneous assembly when its concentration is above critical micelle concentration (CMC). They have a number of unique features, including nanosize, easy manipulation of surface chemistry, core functionalities,

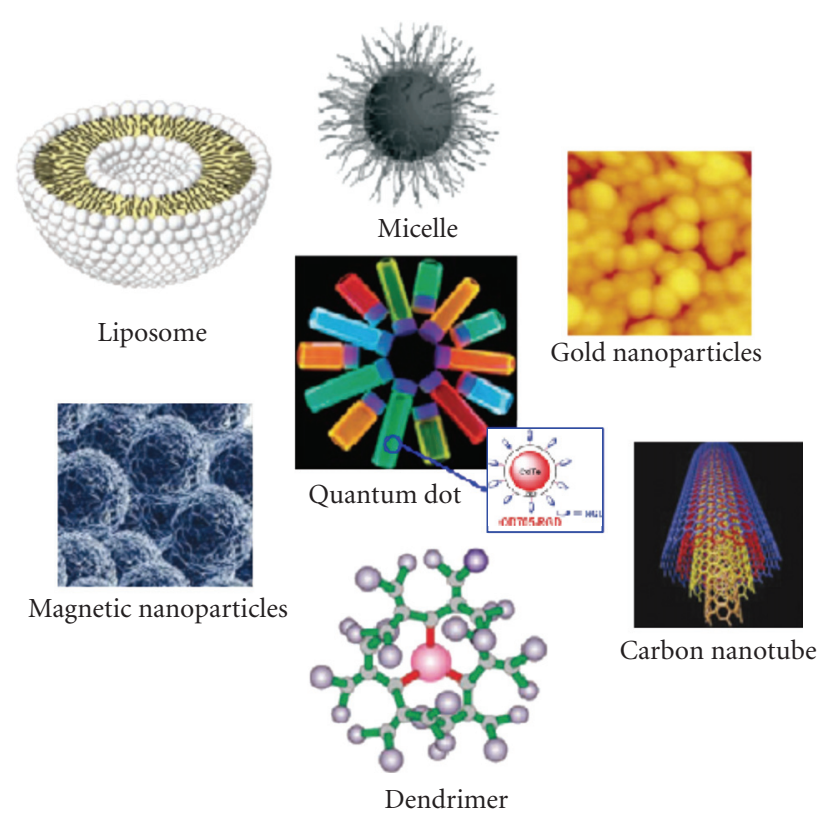

FIgURE 1: Examples of nanomaterials and nanocarrier systems (adapted from $[11,15])$.

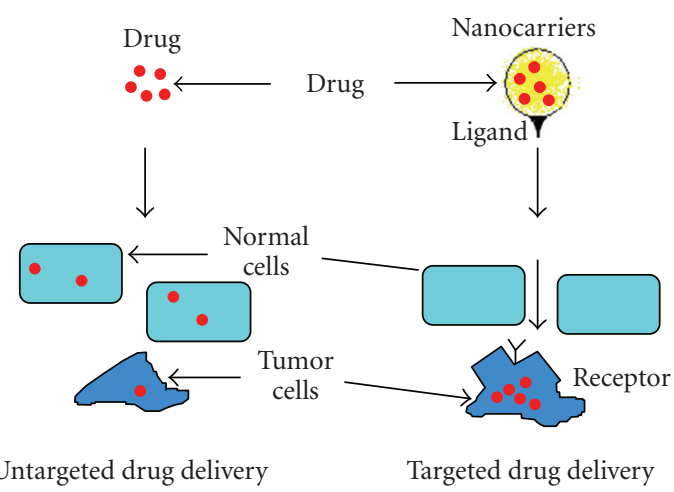

FIGURE 2: Schematic of nanocarrier systems for site-targeted drug delivery (cited from [16]).

as well as ease of fabrication, making them suitable as carriers for encapsulation, and delivery of water insoluble agents [4]. The micelles have a solid-like inner core, which serves as a potent nanocontainer of hydrophobic compounds for solubilization of chemotherapeutics, including docetaxel (DOC) [20], paclitaxel (PTX) [21], camptothecin [22], and dequalinium (DQA) [23]. While polyionic complex (PIC) micelles and cationic polymer micelles can incorporate and protect anionic gene or protein with low rate of cellular uptake and low physiological environment stability, such as vascular endothelial growth factor (VEGF), siRNA [24], and luciferase reporter gene [25]. Thanks to their hydrophilic shell, polymer micelles play an important part in escaping the recognition of RES and prolonging the blood circulation of drugs. The small size $(<100 \mathrm{~nm})$ allows micelles for efficient accumulation in pathological tissues with permeabilized vasculature via the enhanced permeability and retention (EPR) 


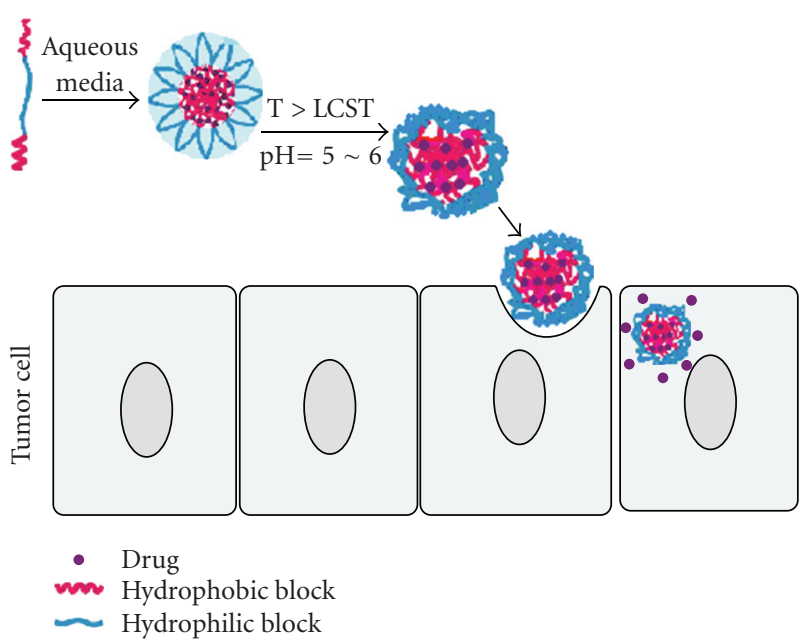

Figure 3: Schematic of drug release from micelles with temperature or $\mathrm{pH}$ trigger when they are uptaken by tumor cells (adapted from $[35])$.

effect [26]. However, the physiological factors, such as the density and heterogeneity of the vasculature at tumor sites, interstitial fluid pressure, and transport of macromolecules in the tumor interstitium, are responsible for the extent of micelles extravasations. Mikhail contributed a detailed review in this perspective [27].

Stimuli-responsive polymeric micelles are often designed for controlled release of drug into tumor tissue with external stimuli trigger, like temperature, $\mathrm{pH}$, ultrasound, and special enzymes $[28,29]$ (Table 1). Among these stimuli, $\mathrm{pH}$ and temperature are of representativeness, because the external $\mathrm{pH}$ of cancerous tissue tends to be lower and the temperature is higher compared to the surrounding normal tissue, which are caused by abnormal metabolism of cancer tissues [4]. Lower critical solution temperature (LCST) polymers, such as poly ( $\mathrm{N}$-isopropylacrylamide) (PNIPAAm) with a cloud point around $32^{\circ} \mathrm{C}$ or some other poly (N-alkylacrylamide) compounds, were investigated as components of temperature-responsive copolymer micelles [29]. The micelles exhibited rapid and temperatureresponsive drug release in cancer cells, which was caused by the destruction of the hydrophobic-hydrophilic balance with the increase of temperature (Figure 3). Licciardi et al. synthesized novel folic acid- (FA-) functionalized diblock copolymer micelles for target delivery of antitumor drugs. The micelles could be directly targeted delivery via folic acid and rapidly release of drug could be triggered by lowering the solution $\mathrm{pH}$ to 5 . This strategy combined the targeted delivery of therapeutics and $\mathrm{pH}$-controlled drug release together, providing a tumor-selective nanocarrier for the efficient delivery of anticancer drugs [30].

2.3. Dendrimers. Dendrimers are artificial macromolecules with tree-like structures in which the atoms are arranged in many branches and subbranches radiate out from a central core $[36,37]$. They are synthesized from branched monomer units in a stepwise manner (Figure 4). Thus it is possible to control their molecular properties, such as size, shape, dimension, and polarity, which depend on the branched monomer units [37]. These highly branched architectures offer unique interfacial and functional performance advantages due to their empty internal cavities and surface functional groups [38]. So they have an enormous capacity for solubilization of hydrophobic drugs and can be modified or conjugated with various interesting guest molecules [37]. Based on the specific properties, the dendrimers have shown great promise in the development of anticancer drug delivery systems [39].

To achieve active targeting drugs to tumor tissues, the well-defined multivalency of dendrimers are widely exploited for covalent attachment of special targeting moieties, such as sugar [40], folic acid [30], antibody [41], biotin [37], and epidermal growth factors [42]. At the same time, therapeutic drugs can be encapsulated into or conjugated with dendrimers. For example, Choi and coworkers have prepared generation 5 polyamidoamine (G5 PAMAM) dendrimers conjugated to fluorescein and folic acid, and then linked them together using complementary DNA oligonucleotides to produce clustered molecules for targeting cancer cells that overexpress the high-affinity folate receptor. In vitro studies indicated the DNA-linked dendrimer clusters could specifically bind to $\mathrm{KB}$ cells and may be used as imaging agents and therapeutics for cancer therapy [43].

2.4. Carbon Nanotubes. Carbon nanotubes are cylinders of one several coaxial graphite layers with a diameter in the order of nanometers, and they serve as instructive examples of the Janus-like properties of nanomaterials [44]. They can be classified into two general categories based on their structure: single-walled carbon nanotubes (SWCNTs) with a single cylindrical carbon wall and multiwalled carbon nanotubes (MWCNTs) with multiple walls-cylinders nested within other cylinders [45] (Figure 5). Thanks to their unique electronic, thermal, and structural characteristics, they can offer a promising approach for gene and drug delivery for cancer therapy $[16,45]$.

Heating of organs and tissues by placing multifunctional nanomaterials at tumor sites is emerging as an art of tumor treatment by "nanothermal therapy" [46]. Carbon nanotubes have become candidates to kill cancer cells via local hyperthermia, due to their thermal conductivity and optical properties. A research showed that oligonucleotides could be translocated into cell nucleus by nanotubes and cause cell death with continuous near-infrared radiation (NIR) because of excessive local heating of SWCNT in vitro [47]. It can afford carbon nanotubes an opportunity to be uptaken only by cancerous cells via functionalization of them with tumor-specific ligands and antibody, like folic acid and monoclonal antibody which can act as targeting agents for many tumors [48]. Accordingly, a highly effective drug delivery system triggered by $\mathrm{pH}$ change has been developed via firstly coated with a polysaccharide material and then modified with folic acid [48]. The encapsulated doxorubicin was only released from the modified nanotubes at a low $\mathrm{pH}$, resulting in nuclear DNA damage and the Hela 
TABLE 1: Stimuli that can be utilized to control the behavior and properties of micelles.

\begin{tabular}{lll}
\hline Stimuli & Stimuli origin & Examples \\
\hline $\mathrm{pH}$ & $\begin{array}{l}\text { Decreased } \mathrm{pH} \text { in cancer site } \\
\text { caused by hypoxia and massive } \\
\text { cell death }\end{array}$ & $\begin{array}{l}\text { Tumoral acidic extracellular pH } \\
\text { targeting of pH-responsive } \\
\text { micelles encapsulated } \\
\text { doxorubicin for cancer therapy }\end{array}$ \\
\hline Temperature & $\begin{array}{l}\text { Increased temperature in } \\
\text { cancerous tissues owing to their } \\
\text { high metabolic activities. } \\
\text { External applied heating }\end{array}$ & $\begin{array}{l}\text { Self-assembled, thermosensitive } \\
\text { micelles for controlled } \\
\text { doxorubicin delivery }\end{array}$ \\
\hline Magnetic field & Locally applied magnetic field & $\begin{array}{l}\text { Folate-encoded and } \\
\text { [3e } \text { On }_{4} \text {-loaded polymeric micelles } \\
\text { for dual targeting of cancer cells }\end{array}$ \\
\hline Ultrasound & Locally applied ultrasound & $\begin{array}{l}\text { Ultrasonic release of doxorubicin } \\
\text { from pluronic P105 micelles }\end{array}$ \\
\hline & $\begin{array}{l}\text { Increased concentration of } \\
\text { glutathione inside many } \\
\text { cancerous cells }\end{array}$ & $\begin{array}{l}\text { Poly(ethylene glycol)-modied } \\
\text { thiolated gelatin nanoparticles } \\
\text { for glutathione-responsive } \\
\text { intracellular DNA delivery }\end{array}$ \\
\hline
\end{tabular}

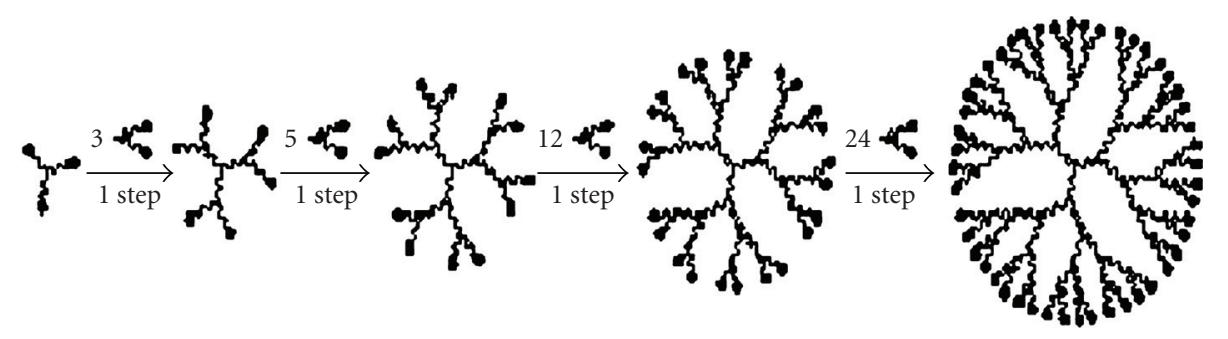

FIGURE 4: Dendrimers synthesized in a stepwise manner (cited from [38]).

cell proliferation inhibition. However, the pharmacokinetics, biodistribution, toxicity, and activity of novel functional carbon nanotubes in vivo should be further investigated to ensure their future applications [46].

2.5. Quantum Dots (QDs). Quantum dots are inorganic fluorescent semiconductor nanoparticels composed of 1050 atoms with a diameter ranging from 2 to $10 \mathrm{~nm}[11,49]$. Their sizes and shapes which determine their absorption and emission properties can be controlled precisely [36]. They are widely studied for optical image application in living systems and are stable for months without degradation and alteration [11]. Targeted ligands have been attached to QDs in order to achieve specific targeting for tumor cell labeling [49]. Thus, they are assured to be chosen as long-term, high-sensitivity and multicontrast imaging agents applied for the detection and diagnosis of cancer in vivo [36].

Now, many researchers focus on using quantum dots as carriers for genes delivery to overcome the obstacles of cell membranes. Klein and coworkers have developed functionalized silicon quantum dots (SiQDs) to serve as self-tracking transfection tool for ABCB1 siRNA [50]. Li et al. investigated glutathione-mediated release of functional plasmid DNA from positively charged CdTe quantum dots, which suggested potential applications of these QDs in selective unpacking of payload in living cells in a visible manner [51]. The applicability of quantum dots for cancer therapies based on the mechanisms of photosensitization and radiosensitization has also been investigated. Possessing electronic energy levels in the range of $1-5 \mathrm{eV}$, quantum dots can perform as photosentizers applied in photodynamic therapy (PDT), which has recently become an approved treatment modality for some type of cancer. Due to high atom and electron density, quantum dots could absorb highenergy photons acting as radiosensitizers to cause localized and targeted damages to cancer cells, which was reviewed in details by Juzenas [52].

With the help of near-infrared (NIR) optical imaging devices, QDs-based tumor imaging and treatment could allow for application in deeper tissues and may offer optical guide for surgery on organs. However, the toxicity of QDs cannot be ignored for their applications in vivo, because they are composed of hazardous heavy metals. Accordingly, it is necessary to investigate their toxicity systematically to ensure their security for further applications to human.

2.6. Nanofibers. Electrospinning has gained widespread interest as a potential polymer processing technique to produce ultrafine polymer fibers for drug delivery applications. It has been proven to be a relatively simple and versatile method for producing polymeric fibers with diameters ranging from tens of nanometers to microns [53]. 


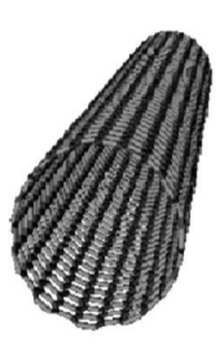

SWCNT

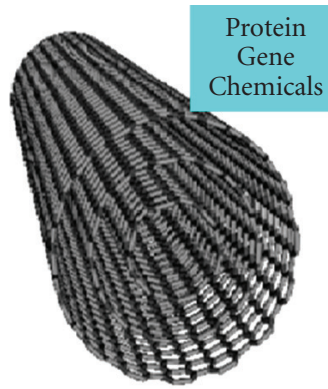

MWCNT

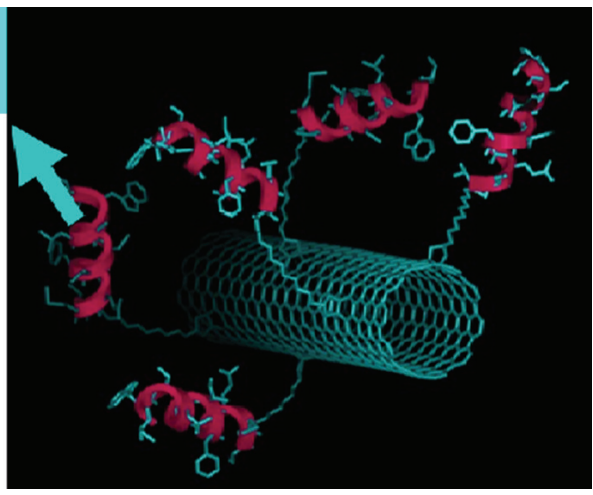

Figure 5: Structure of single-walled carbon nanotubes (SWCNTs) and multiwalled carbon nanotubes (MWCNTs), as well as drug loaded carbon nanotubes (adapted from http://www-ibmc.u-strasbg.fr/ict/vectorisation/nanotubes_eng.shtml and http://www-ibmc.u-strasbg .fr/ict/vectorisation/vectorisation_eng.shtml).

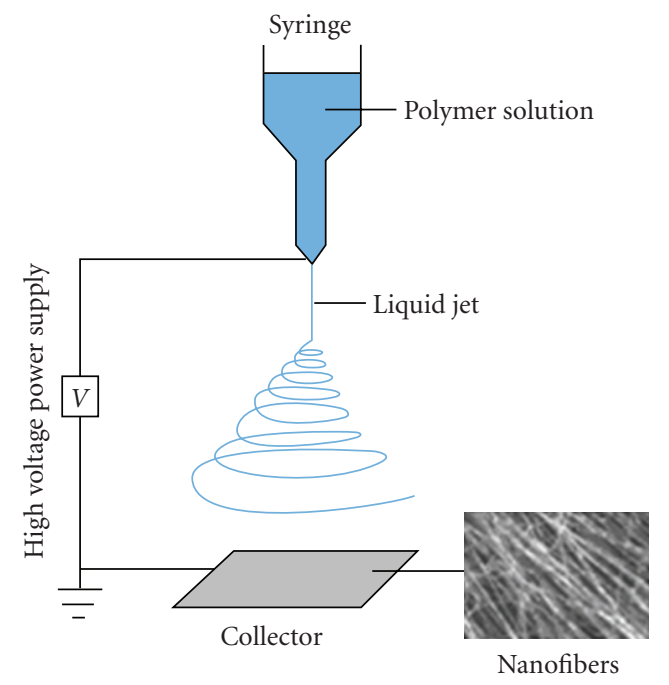

Figure 6: Schematic of electrospinning process (adapted from [56]).

Electrospinning process can be briefly described as follows. A polymer solution is ejected from the capillary outlet of a syringe by strong enough electrostatic forces, and finally deposits as a nonwoven fabric mat on a grounded metal screen (counter electrode). As this jet travels through the air, the solvent evaporates, leaving behind ultrafine polymer fibers [54, 55] (Figure 6). Accordingly, the properties of nanofibers can be controlled easily by a number of processing parameters, such as applied voltage, polymer flow rate, capillary-collector distance, as well as the surface tension and viscoelasticity of solution [53].

As a fibrous scaffold, nanofibers are able to entrap drugs with a high loading capacity and high encapsulation efficiency because of their low weight and inherent high surfaceto-volume ratio. They have been designed as promising carriers for delivering anticancer drugs, especially in postoperative local chemotherapy via surgical implantation of the scaffold [57]. Xie and Wang develop electrospun PLGAbased micro- and nanofibers as implants for the sustained delivery of pacilitaxel to treat C6 glioma in vitro. With an increased drug loading and prolonged release period, the antitumor efficiency was comparable to commercial pacilitaxel formulation Taxol [58]. Xu et al. developed implantable BCNU-loaded poly (ethylene glycol)-poly (L-lactic acid) (PEG-PLLA) diblock copolymer fibers for the controlled release of 1,3-bis (2-chloroethyl)-1-nitrosourea (BCNU) for postoperative chemotherapy of cancers. Compared with pristine BCUN, the BCNU released from the fiber retained its efficacy for prolonged period and increased antitumor activity against rat Glioma C6 cells [59]. Recently, they prepared nanofibers entrapped both paclitaxel (PTX) and doxorubicin hydrochloride (DOX) by means of "emulsionelectrospinning," providing a new approach for multidrug delivery on combination therapy [60]. Although electrospun nanofibers have been investigated greatly and show effective antitumor activity as drug carriers for postoperative local chemotherapy in vitro, further researches on the destiny and antitumor activity of the nanofibers in vivo should be investigated.

2.7. Others. Current nanotechnology platforms for cancer therapeutics also include other nanomaterials. In addition to polymeric nanoparticles, gold nanoparticles (GNPs) and magnetic nanoparticles are being paid more attention because their unique physical, chemical, and biological properties are quite different from the bulk of their counterparts [7]. They can act as active medicine or drug additives for detection and hyperthermia treatment of cancer after laser irradiation [65, 66]. Nanoshells which consist of a silica core and a thin gold shell are designed to apply in cancer imaging and therapy. Because of the silica core and gold shell, the most useful nanoshells can strongly absorb NIR light and create intense heat that is lethal to cells for thermal ablation therapy [67]. Recombinant adenovirus (Ad) can produce a linear, double-stranded viral genome that does not integrate with the genome of the host cell during the replication but induces a DNA double-strand break repair response in the host cell. There are many studies on the 
TABLE 2: Advantages of nanotechnology-based on drug delivery systems in cancer therapy.

\begin{tabular}{ll}
\hline Features & Examples \\
\hline Solubilization effect & $\begin{array}{l}\text { Self-assembled polymeric micelles with hydrophobic core can serve as a } \\
\text { potent nanocontainer for increasing the solubility of hydrophobic drugs. }\end{array}$ \\
\hline Protective effect & $\begin{array}{l}\text { Entrapment or conjugation of a drug to polymeric systems may protect the } \\
\text { drug from inactivation and help to store its activity for prolonged duration. }\end{array}$ \\
\hline & $\begin{array}{l}\text { The enhanced permeability and retention (EPR) effect allows for the } \\
\text { accumulation of drug carriers in the interstitial fluid of the diseased tissue. } \\
\text { The drug entrapped or conjugated to the carriers can be retained in the } \\
\text { tumor tissue for a longer time, whereas free drug with low-molecular } \\
\text { Peight easily diffuse back out. }\end{array}$ \\
\hline Active targeting & $\begin{array}{l}\text { Active targeting can be achieved via specific recognition processes, } \\
\text { including ligand-receptor and antibody-antigen recognition by the surface } \\
\text { modification with various ligands, such as folate, mannose, and galactose. }\end{array}$ \\
\hline Controlled release of drugs & $\begin{array}{l}\text { The drug can be controlled release at diseased site when an appropriate } \\
\text { signal is offered, such as pH, temperature, ultrasound, or special enzymes. }\end{array}$ \\
\hline
\end{tabular}

use of recombinant adenovirus particles as carriers for sensitizers to enhance noninvasive cancer therapeutics, such as radiation therapy, photodynamic therapy, and photothermal therapy. They can also be fabricated to combine multiple functions of targeting, imaging, and drug delivery together to increase the sensitivity and specificity of cancer therapy [68].

\section{Summary}

Nanotechnology-based drug carriers and materials have yielded more medical benefits in the recent years, especially the field of cancer therapy. Compared to conventional formations, nanocarrier systems have many advantages. For example, they can improve the solubility of poorly soluble drugs [20], protect the recombinant of protein and genes [61], circulate in blood stream for longer time without being recognized by macrophages, as well as controlled release of drugs at an expected rate in the desired area [28] (Table 2). Due to their various advantages, the nanocarrier systems and nanomaterials have demonstrated comparable or superior anticancer efficiency to commercial formations and provided new strategies to fight against cancer.

\section{Problems and Future Perspective}

The nanoscale platforms have made significant progress in formulation preparations and achieved more precise treatment at a molecular level. There are already novel formations for cancer therapy available commercially, such as Abraxane, Doxil, and Embosphere, et al. [69-71], and they may offer great opportunities for personalized medicine. Although drug delivery systems and nanomaterials are mostly investigated in preclinical animal models, too few studies are carried out to examine the cellular uptake of delivery systems in human body, because (a) the data from pre-clinical animals cannot exactly reflect its effect on human body because of the complexity of tumor biology and the differences between animal and human physiology, (b) the development of new drug delivery systems is based on the advancement of the carrier materials, but there are only few types of materials approved by Food and Drug Administration (FDA), and (c) the security and the longterm effects of nanotechnology are unknown, which need research strategies to evaluate the specific risks when used in human body. With the development of cancer biology and polymer chemistry, new nanotechnology-based tools and therapeutic strategies will be designed and applied in the fields of cancer precautiondiagnosis and treatment. Besides, nanotechnology also plays an important part in delivery of drugs for ocular therapy and in generation of scaffolds for tissue engineering. However, the other major developments will have to take place for them to be permanently established not only in academia but also in industry. The basic theories of drug release from nanocarriers and pharmacokinetic models need to be developed and improved. The cytotoxicity of nanomedicine or their degradation products remains a serious problem, so more attention should be paid to improve the biocompatibility in future investigations. Their toxicity, long-term stability, and degradation pathways may also influence the integrity of the environment. It will not be of great benefit to clinical application and industrial production of nanomedicine unless the problems are solved.

\section{Acknowledgment}

The authors would like to thank Dr. Gurinder K. Singh for her critical evaluation and helpful suggestions to the paper.

\section{References}

[1] F. H. Sarkar, S. Banerjee, and Y. W. Li, "Pancreatic cancer: pathogenesis, prevention and treatment," Toxicology and Applied Pharmacology, vol. 224, no. 3, pp. 326-336, 2007.

[2] J. D. Byrne, T. Betancourt, and L. Brannon-Peppas, "Active targeting schemes for nanoparticle systems in cancer therapeutics," Advanced Drug Delivery Reviews, vol. 60, no. 15, pp. 1615-1626, 2008. 
[3] A. K. Iyer, G. Khaled, J. Fang, and H. Maeda, "Exploiting the enhanced permeability and retention effect for tumor targeting," Drug Discovery Today, vol. 11, no. 17-18, pp. 812818, 2006.

[4] N. Wiradharma, Y. Zhang, S. Venkataraman, J. L. Hedrick, and Y. Y. Yang, "Self-assembled polymer nanostructures for delivery of anticancer therapeutics," Nano Today, vol. 4, no. 4, pp. 302-317, 2009.

[5] L. Jabr-Milane, L. V. Vlerken, H. Devalapally, et al., "Multifunctional nanocarriers for targeted delivery of drugs and genes," Journal of Controlled Release, vol. 130, no. 2, pp. 121$128,2008$.

[6] M. Pulkkinen, J. Pikkarainen, T. Wirth, et al., "Threestep tumor targeting of paclitaxel using biotinylated PLAPEG nanoparticles and avidin-biotin technology: formulation development and in vitro anticancer activity," European Journal of Pharmaceutics and Biopharmaceutics, vol. 70, no. 1, pp. 66-74, 2008.

[7] N. R. Panyala, E. M. Peña-Méndez, and J. Havel, "Gold and nano-gold in medicine: overview, toxicology and perspectives," Journal of Applied Biomedicine, vol. 7, no. 2, pp. 75-91, 2009.

[8] K. K. Jain, "Nanotechnology in clinical laboratory diagnostics," Clinica Chimica Acta, vol. 358, no. 1-2, pp. 37-54, 2005.

[9] G. Linazasoro, "Potential applications of nanotechnologies to Parkinson's disease therapy," Parkinsonism and Related Disorders, vol. 14, no. 5, pp. 383-392, 2008.

[10] W. Cai, A. R. Hsu, Z.-B. Li, and X. Chen, "Are quantum dots ready for in vivo imaging in human subjects?" Nanoscale Research Letters, vol. 2, no. 6, pp. 265-281, 2007.

[11] W. Cai and X. Chen, "Nanoplatforms for targeted molecular imaging in living subjects," Small, vol. 3, no. 11, pp. 1840 $1854,2007$.

[12] A. K. Bajpai, S. K. Shukla, S. Bhanu, and S. Kankane, "Responsive polymers in controlled drug delivery," Progress in Polymer Science, vol. 33, no. 11, pp. 1088-1118, 2008.

[13] C. Fonseca, S. Simoes, and R. Gaspar, "Paclitaxel-loaded PLGA nanoparticles: preparation, physicochemical characterization and in vitro anti-tumoral activity," Journal of Controlled Release, vol. 83, no. 2, pp. 273-286, 2002.

[14] M. L. Hans and A. M. Lowman, "Biodegradable nanoparticles for drug delivery and targeting," Current Opinion in Solid State and Materials Science, vol. 6, no. 4, pp. 319-327, 2002.

[15] N. Sanvicens and M. P. Marco, "Multifunctional nanoparticles-properties and prospects for their use in human medicine," Trends in Biotechnology, vol. 26, no. 8, pp. 425-433, 2008.

[16] T. Tanaka, S. Shiramoto, M. Miyashita, Y. Fujishima, and Y. Kaneo, "Tumor targeting based on the effect of enhanced permeability and retention (EPR) and the mechanism of receptor-mediated endocytosis (RME)," International Journal of Pharmaceutics, vol. 277, no. 1-2, pp. 39-61, 2004.

[17] S. Sandhiya, S. A. Dkhar, and A. Surendiran, "Emerging trends of nanomedicine-an overview," Fundamental and Clinical Pharmacology, vol. 23, no. 3, pp. 263-269, 2009.

[18] Y. Fukumori and H. Ichikawa, "Nanoparticles for cancer therapy and diagnosis," Advanced Powder Technology, vol. 17, no. 1, pp. 1-28, 2006.

[19] M. Johannsen, U. Gneveckow, B. Thiesen, et al., "Thermotherapy of prostate cancer using magnetic nanoparticles: feasibility, imaging, and three-dimensional temperature distribution," European Urology, vol. 52, no. 6, pp. 1653-1662, 2007.
[20] B. Liu, M. Yang, R. Li, et al., "The antitumor effect of novel docetaxel-loaded thermosensitive micelles," European Journal of Pharmaceutics and Biopharmaceutics, vol. 69, no. 2, pp. 527534, 2008.

[21] K. M. Huh, H. S. Min, S. C. Lee, H. J. Lee, S. Kim, and K. Park, "A new hydrotropic block copolymer micelle system for aqueous solubilization of paclitaxel," Journal of Controlled Release, vol. 126, no. 2, pp. 122-129, 2008.

[22] O. M. Koo, I. Rubinstein, and H. Onyuksel, "Camptothecin in sterically stabilized phospholipid micelles: a novel nanomedicine," Nanomedicine: Nanotechnology, Biology, and Medicine, vol. 1, no. 1, pp. 77-84, 2005.

[23] C. Lizano, V. Weissig, V. P. Torchilin, P. Sancho, A. I. García-Pérez, and M. Pinilla, "In vivo biodistribution of erythrocytes and polyethyleneglycol-phosphatidylethanolamine micelles carrying the antitumour agent dequalinium," European Journal of Pharmaceutics and Biopharmaceutics, vol. 56, no. 2, pp. 153-157, 2003.

[24] A. M. Al-Abd, S. H. Lee, S. H. Kim, et al., "Penetration and efficacy of VEGF siRNA using polyelectrolyte complex micelles in a human solid tumor model in-vitro," Journal of Controlled Release, vol. 137, no. 2, pp. 130-135, 2009.

[25] N. Wiradharma, Y. W. Tong, and Y. -Y. Yang, "Self-assembled oligopeptide nanostructures for co-delivery of drug and gene with synergistic therapeutic effect," Biomaterials, vol. 30, no. 17, pp. 3100-3109, 2009.

[26] L. B. Li and Y. B. Tan, "Preparation and properties of mixed micelles made of Pluronic polymer and PEG-PE," Journal of Colloid and Interface Science, vol. 317, no. 1, pp. 326-331, 2008.

[27] A. S. Mikhail and C. Allen, "Block copolymer micelles for delivery of cancer therapy: transport at the whole body, tissue and cellular levels," Journal of Controlled Release, vol. 138, no. 3, pp. 214-223, 2009.

[28] G. A. Husseini, N. Y. Rapoport, D. A. Christensen, J. D. Pruitt, and W. G. Pitt, "Kinetics of ultrasonic release of doxorubicin from pluronic P105 micelles," Colloids and Surfaces B, vol. 24, no. 3-4, pp. 253-264, 2002.

[29] N. Rapoport, "Physical stimuli-responsive polymeric micelles for anti-cancer drug delivery," Progress in Polymer Science, vol. 32, no. 8-9, pp. 962-990, 2007.

[30] M. Licciardi, G. Giammona, J. Du, S. P. Armes, Y. Tang, and A. L. Lewis, "New folate-functionalized biocompatible block copolymer micelles as potential anti-cancer drug delivery systems," Polymer, vol. 47, no. 9, pp. 2946-2955, 2006.

[31] J. Ko, K. Park, Y.-S. Kim, et al., "Tumoral acidic extracellular $\mathrm{pH}$ targeting of $\mathrm{pH}$-responsive MPEG-poly( $\beta$-amino ester) block copolymer micelles for cancer therapy," Journal of Controlled Release, vol. 123, no. 2, pp. 109-115, 2007.

[32] M. Nakayama, T. Okano, T. Miyazaki, F. Kohori, K. Sakai, and M. Yokoyama, "Molecular design of biodegradable polymeric micelles for temperature-responsive drug release," Journal of Controlled Release, vol. 115, no. 1, pp. 46-56, 2006.

[33] X. Yang, Y. H. Chen, R. Yuan, et al., "Folate-encoded and $\mathrm{Fe}_{3} \mathrm{O}_{4}$-loaded polymeric micelles for dual targeting of cancer cells," Polymer, vol. 49, no. 16, pp. 3477-3485, 2008.

[34] V. Torchilin, "Multifunctional and stimuli-sensitive pharmaceutical nanocarriers," European Journal of Pharmaceutics and Biopharmaceutics, vol. 71, no. 3, pp. 431-444, 2009.

[35] C. Chang, H. Wei, C.-Y. Quan, et al., "Fabrication of thermosensitive PCL-PNIPAAm-PCL triblock copolymeric micelles for drug delivery," Journal of Polymer Science A, vol. 46, no. 9, pp. 3048-3057, 2008. 
[36] K. J. Morrow Jr., R. Bawa, and C. Wei, "Recent advances in basic and clinical nanomedicine," Medical Clinics of North America, vol. 91, no. 5, pp. 805-843, 2007.

[37] W. J. Yang, Y. Y. Cheng, T. W. Xu, X. Y. Wang, and L. P. Wen, "Targeting cancer cells with biotine-dendrimer conjugates," European Journal of Medicinal Chemistry, vol. 44, no. 2, pp. 862-868, 2009.

[38] S. Svenson and D. A. Tomalia, "Dendrimers in biomedical applications-reflections on the field," Advanced Drug Delivery Reviews, vol. 57, no. 15, pp. 2106-2129, 2005.

[39] E. R. Gillies and J. M. J. Fréchet, "Dendrimers and dendritic polymers in drug delivery," Drug Discovery Today, vol. 10, no. 1, pp. 35-43, 2005.

[40] D. Bhadra, A. K. Yadav, S. Bhadra, and N. K. Jain, "Glycodendrimeric nanoparticulate carriers of primaquine phosphate for liver targeting," International Journal of Pharmaceutics, vol. 295, no. 1-2, pp. 221-233, 2005.

[41] A. K. Patri, A. Myc, J. Beals, T. P. Thomas, N. H. Bander, and J. R. Baker Jr., "Synthesis and in vitro testing of J591 antibodydendrimer conjugates for targeted prostate cancer therapy," Bioconjugate Chemistry, vol. 15, no. 6, pp. 1174-1181, 2004.

[42] M. Hussain, M. Shchepinov, M. Sohail, et al., "A novel anionic dendrimer for improved cellular delivery of antisense oligonucleotides," Journal of Controlled Release, vol. 99, no. 1, pp. 139-155, 2004.

[43] Y. Choi, T. Thomas, A. Kotlyar, M. T. Islam, and J. R. Baker Jr., "Synthesis and functional evaluation of DNA-assembled polyamidoamine dendrimer clusters for cancer cell-specific targeting," Chemistry and Biology, vol. 12, no. 1, pp. 35-43, 2005.

[44] A. A. Shvedova, E. R. Kisin, D. Porter, et al., "Mechanisms of pulmonary toxicity and medical applications of carbon nanotubes: two faces of Janus?" Pharmacology and Therapeutics, vol. 121, no. 2, pp. 192-204, 2009.

[45] L. Lacerda, A. Bianco, M. Prato, and K. Kostarelos, "Carbon nanotubes as nanomedicines: from toxicology to pharmacology," Advanced Drug Delivery Reviews, vol. 58, no. 14, pp. 1460-1470, 2006.

[46] R. Sharma and C. J. Chen, "Newer nanoparticles in hyperthermia treatment and thermometry," Journal of Nanoparticle Research, vol. 11, no. 3, pp. 671-689, 2009.

[47] N. W. S. Kam, M. O'Connell, J. A. Wisdom, and H. Dai, "Carbon nanotubes as multifunctional biological transporters and near-infrared agents for selective cancer cell destruction," Proceedings of the National Academy of Sciences of the United States of America, vol. 102, no. 33, pp. 11600-11605, 2005.

[48] X. K. Zhang, L. J. Meng, Q. H. Lu, Z. F. Fei, and P. J. Dyson, "Targeted delivery and controlled release of doxorubicin to cancer cells using modified single wall carbon nanotubes," Biomaterials, vol. 30, no. 30, pp. 6041-6047, 2009.

[49] K. Y. Kim, "Nanotechnology platforms and physiological challenges for cancer therapeutics," Nanomedicine: Nanotechnology, Biology, and Medicine, vol. 3, no. 2, pp. 103-110, 2007.

[50] S. Klein, O. Zolk, M. F. Fromm, F. Schrödl, W. Neuhuber, and C. Kryschi, "Functionalized silicon quantum dots tailored for targeted siRNA delivery," Biochemical and Biophysical Research Communications, vol. 387, no. 1, pp. 164-168, 2009.

[51] D. Li, G. P. Li, W. Guo, P. Li, E. Wang, and J. Wang, "Glutathione-mediated release of functional plasmid DNA from positively charged quantum dots," Biomaterials, vol. 29, no. 18, pp. 2776-2782, 2008.

[52] P. Juzenas, W. Chen, Y.-P. Sun, et al., "Quantum dots and nanoparticles for photodynamic and radiation therapies of cancer," Advanced Drug Delivery Reviews, vol. 60, no. 15, pp. 1600-1614, 2008.

[53] M. Wang, A. J. Hsieh, and G. C. Rutledge, "Electrospinning of poly(MMA-co-MAA) copolymers and their layered silicate nanocomposites for improved thermal properties," Polymer, vol. 46, no. 10, pp. 3407-3418, 2005.

[54] X. L. Xu, X. S. Chen, A. X. Liu, Z. K. Hong, and X. B. Jing, "Electrospun poly(L-lactide)-grafted hydroxyapatite/ poly(Llactide) nanocomposite fibers," European Polymer Journal, vol. 43, no. 8, pp. 3187-3196, 2007.

[55] M. Wang, H. Singh, T. A. Hatton, and G. C. Rutledge, "Field-responsive superparamagnetic composite nanofibers by electrospinning," Polymer, vol. 45, no. 16, pp. 5505-5514, 2004.

[56] E. Jo, S. Lee, K. T. Kim, et al., "Core-sheath nanofibers containing colloidal arrays in the core for programmable multi-agent delivery," Advanced Materials, vol. 20, no. 9, pp. 968-972, 2008.

[57] X. Xu, X. Chen, P. Ma, X. Wang, and X. Jing, "The release behavior of doxorubicin hydrochloride from medicated fibers prepared by emulsion-electrospinning," European Journal of Pharmaceutics and Biopharmaceutics, vol. 70, no. 1, pp. 165170, 2008.

[58] J. Xie and C.-H. Wang, "Electrospun micro- and nanofibers for sustained delivery of paclitaxel to treat C6 Glioma in vitro," Pharmaceutical Research, vol. 23, no. 8, pp. 1817-1826, 2006.

[59] X. Xu, X. Chen, X. Xu, et al., "BCNU-loaded PEG-PLLA ultrafine fibers and their in vitro antitumor activity against Glioma C6 cells," Journal of Controlled Release, vol. 114, no. 3, pp. 307-316, 2006.

[60] X. L. Xu, X. S. Chen, Z. F. Wang, and X. B. Jing, "Ultrafine PEG-PLA fibers loaded with both paclitaxel and doxorubicin hydrochloride and their in vitro cytotoxicity," European Journal of Pharmaceutics and Biopharmaceutics, vol. 72, no. 1, pp. 18-25, 2009.

[61] Y. T. Ko, A. Kale, W. C. Hartner, B. PapahadjopoulosSternberg, and V. P. Torchilin, "Self-assembling micellelike nanoparticles based on phospholipid-polyethyleneimine conjugates for systemic gene delivery," Journal of Controlled Release, vol. 133, no. 2, pp. 132-138, 2009.

[62] K. F. Pirollo and E. H. Chang, "Does a targeting ligand influence nanoparticle tumor localization or uptake?" Trends in Biotechnology, vol. 26, no. 10, pp. 552-558, 2008.

[63] D. J. Betting, X. Y. Mu, K. Kafi, et al., "Enhanced immune stimulation by a therapeutic lymphoma tumor antigen vaccine produced in insect cells involves mannose receptor targeting to antigen presenting cells," Vaccine, vol. 27, no. 2, pp. 250-259, 2009.

[64] E. Gabano, M. Ravera, C. Cassino, S. Bonetti, G. Palmisano, and D. Osella, "Stepwise assembly of platinum-folic acid conjugates," Inorganica Chimica Acta, vol. 361, no. 5, pp. 1447$1455,2008$.

[65] H. F. Liang, C. T. Chen, S. C. Chen, et al., "Paclitaxelloaded poly(g-glutamic acid)-poly(lactide) nanoparticles as a targeted drug delivery system for the treatment of liver cancer," Biomaterials, vol. 27, no. 9, pp. 2051-2059, 2006.

[66] L. Zhu, Z. Huo, L. Wang, X. Tong, Y. Xiao, and K. Ni, "Targeted delivery of methotrexate to skeletal muscular tissue by thermosensitive magnetoliposomes," International Journal of Pharmaceutics, vol. 370, no. 1-2, pp. 136-143, 2009.

[67] K. Y. Kim, "Nanotechnology platforms and physiological challenges for cancer therapeutics," Nanomedicine: Nanotechnology, Biology, and Medicine, vol. 3, no. 2, pp. 103-110, 2007. 
[68] R. Singh and K. Kostarelos, "Designer adenoviruses for nanomedicine and nanodiagnostics," Trends in Biotechnology, vol. 27, no. 4, pp. 220-229, 2009.

[69] W. J. Gradishar, S. Tjulandin, N. Davidson, et al., "Phase III trial of nanoparticle albumin-bound paclitaxel compared with polyethylated castor oil-based paclitaxel in women with breast cancer," Journal of Clinical Oncology, vol. 23, no. 31, pp. 7794$7803,2005$.

[70] T. Chidiac, G. T. Budd, R. Pelley, et al., "Phase II trial of liposomal doxorubicin (Doxil ${ }^{\circledR}$ ) in advanced soft tissue sarcomas," Investigational New Drugs, vol. 18, no. 3, pp. 253259, 2000.

[71] S. O. Rodiek, A. Stolzle, and C. B. Lumenta, "Preoperative embolization of intracranial meningiomas with Embosphere ${ }^{\circledR}$ microspheres," Minimally Invasive Neurosurgery, vol. 47, no. 5, pp. 299-305, 2004. 

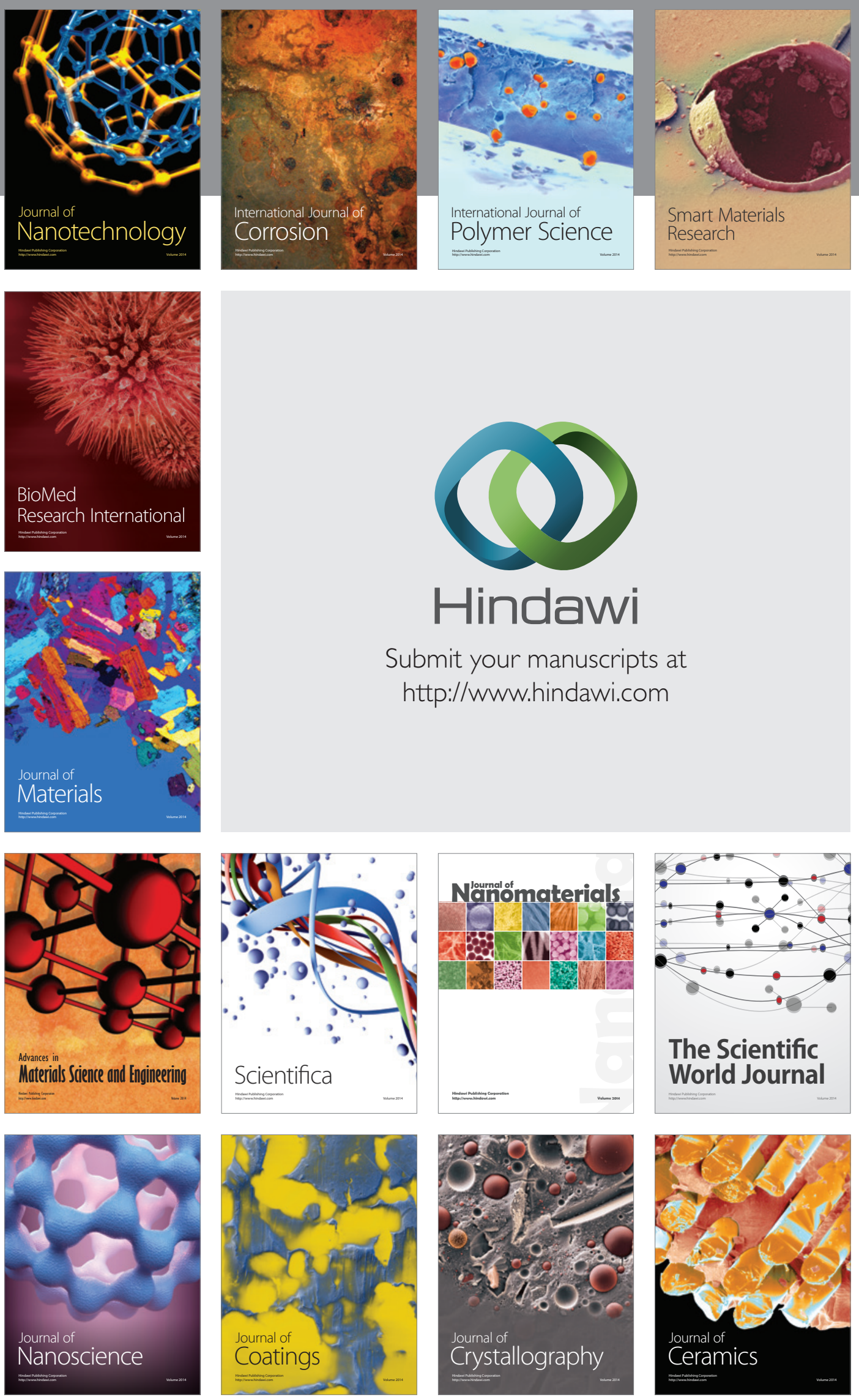

The Scientific World Journal

Submit your manuscripts at

http://www.hindawi.com

\section{World Journal}

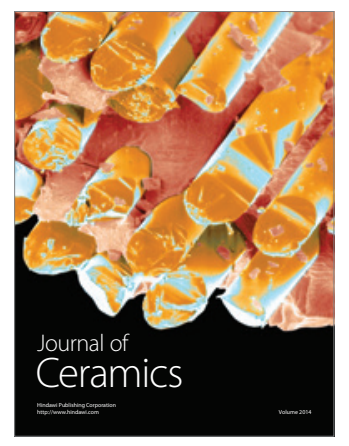

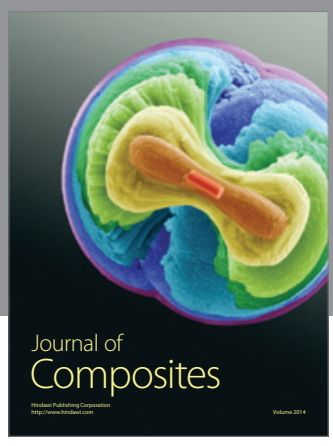
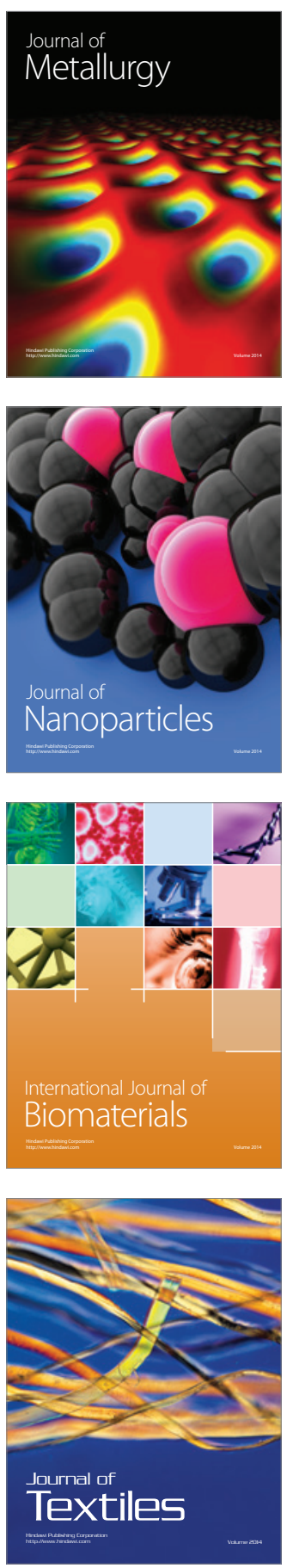